\title{
Impact of the COVID-19 pandemic on surgical skills training in pediatric surgery residents
}

\author{
Luciana Lerendegui $^{1} \cdot$ Rocío Boudou $^{1} \cdot$ Carolina Percul $^{1} \cdot$ Alejandra Curiel $^{1} \cdot$ Eduardo Durante $^{2}$. Juan M. Moldes ${ }^{1}$. \\ Francisco de Badiola ${ }^{1}$ - Daniel H. Liberto ${ }^{1}$. Erica Delorenzi ${ }^{2}$. Pablo A. Lobos ${ }^{1}$
}

Accepted: 30 June 2021 / Published online: 16 July 2021

(c) The Author(s), under exclusive licence to Springer-Verlag GmbH Germany, part of Springer Nature 2021

\begin{abstract}
Purpose To analyze the initial impact of the SARS-CoV-2 pandemic on surgical skills training and performance of Pediatric Surgery Residents.

Methods Retrospective study considering the modifications on the Pediatric Surgery Residency training from March 1st-May 31st, 2020. Exposure to OR learning opportunities was compared to the same 2018-2019 trimesters. An anonymous survey about self-perception on surgical skills development was also performed.

Results Residents performed 209 procedures as leading surgeons during the 2020 trimester with a mean number of surgeries per resident of 20.9, representing a reduction of $46 \%$ and 56.8\% compared to the 2018-2019 averages, respectively. Reduction in both the number and the percentage of total procedures $(n: 209,56.8 \%)$ compared to both $2019(n: 354,68.7 \%, p$ : $0.000272)$ and $2018(n: 420,76.1 \%, p<0,00,001)$ showed statistical correlation with no changes in their complexity pattern. From the survey (response rate: $100 \%$ ), hours dedicated to simulation-based training were highly increased. More time was spent studying, but only $60 \%$ achieved better preparation for surgery and $70 \%$ perceived a decrease in surgical confidence. Conclusions Even though the pandemic promoted new teaching strategies and the use of simulation-based training, it drastically reduced "on-the-job" learning opportunities with potential effects on residents' performance and self-confidence during surgery.
\end{abstract}

Keywords Surgical skills $\cdot$ Resident training $\cdot$ Pediatric surgery $\cdot$ COVID-19

\section{Introduction}

In March 2020, the arrival of the SARS-CoV-2 pandemic to our country induced a rapid change in our healthcare system. Residents training programs are based on controlled exposure to professional practice, so they had to also rapidly readapt [1]. The pediatric population is not the most susceptible one to COVID-19, and surgery seems to play a minor role in the care of infected patients. However, both the pediatric surgical department and associated training programs had to

Luciana Lerendegui

luciana.lerendegui@hospitalitaliano.org.ar

1 Division of Pediatric Surgery and Urology, Department of Pediatrics, Hospital Italiano de Buenos Aires, Potosí, 4060, Buenos Aires, Argentina

2 Instituto Universitario Hospital Italiano de Buenos Aires (IUHIBA), Potosí, 4234, Buenos Aires, Argentina find adaptive mechanisms to cope with the new guidelines within a global action program [2,3].

During the SARS-CoV-2 outbreak, the surgical staff (including Residents) was grouped into teams and a schedule was established. Each team carried out one week of active hospital duties after which they had two mandatory isolation weeks at home, performing mostly academic activities such as preparation for surgical cases, studying for exams, or skills training using simulation models. Residents shifts schedule was modified not to stay at the hospital during the night and to be contacted by the active pediatric surgeon only in case of need. All medical team was trained in the use of personal protective equipment (PPE) and general prevention measures. Regarding operating room management, it was determined that surgeries had to be performed by "the most expert surgeon in the case of suspected or confirmed COVID-19 patients", to reduce the exposure time for health personnel [4]. 
Regarding outpatient care, the Telemedicine modality was rapidly incorporated, and in-person clinical interviews were drastically restricted. Inpatient care continued to be followed up by the team assigned for each week.

The present study aims to analyze the initial impact of the SARS-CoV-2 pandemic in the training of surgical skills for the Pediatric Surgery Residents of a University Hospital in Buenos Aires and the adjustment of learning strategies.

\section{Materials and methods}

A descriptive retrospective study was carried out considering the modifications to the Pediatric Surgery Residency training at our institution from March 1st-May 31st, 2020. The research protocol received approval from the Institutional Review Board (CEPIU-reference number 0030-20). PGY-2 to PGY-5 were included in the analysis. PGY-1 were excluded since they were in General Surgery during the study period.

In the first part of the study, the exposure to surgical learning opportunities was analyzed through the data obtained from the surgical procedure records. A comparison between the study period and the same months of the previous 2 years was conducted. Statistical analysis was carried out using the Chi-Square test for categorical data. Results were expressed as a percentage of increase or decrease for each variable. Additionally, the strategies applied to maintain healthcare activity, reduce the risk of contagion among professionals and continue surgical education were identified and analyzed.

The second part of the study included an anonymous survey (supplemental material). Residents were asked to answer seven questions regarding their self-perception on surgical skills learning and other related topics.

\section{Results}

\section{Surgical procedures}

368 surgeries were registered during the period March 1st to May 31st, 2020 (March: 138, April: 112, and May: 118). The surgeries performed in the previous months are represented to contextualize those numbers Fig. 1.

The total number of registered surgeries corresponding to the periods March 1st-May 31st in the years 2015-2020 was 3020 (Table 1). The average number of surgeries calculated for the selected trimester in the period 2015-2019 was 530.4 (scheduled procedures' mean: 300.2 -emergency surgeries' mean: 230.2). Variability in the total number of procedures was identified during the analysis of those years with a peak during 2017, followed by a decrease and stabilization of the numbers in 2018-2019. The number of residents during those 5 years also showed modifications due to changes in the Residency Program and an increase in the resident-peryear admission rate.

We decided to compare 2020 vs. 2018-2019 data due to the steadiness reached in the 2018-2020 period (Table 1). We found a reduction of $31.02 \%$ in the total number of surgical procedures $(49.08 \%$ for scheduled and $8.09 \%$ for emergencies) in the selected trimester. The emergency/scheduled ratio for the 3 month interval analyzed was 1.42 in 2020, due predominantly to the abrupt decrease in the number of scheduled surgeries Fig. 2.

\section{SURGICAL PROCEDURES TREND}

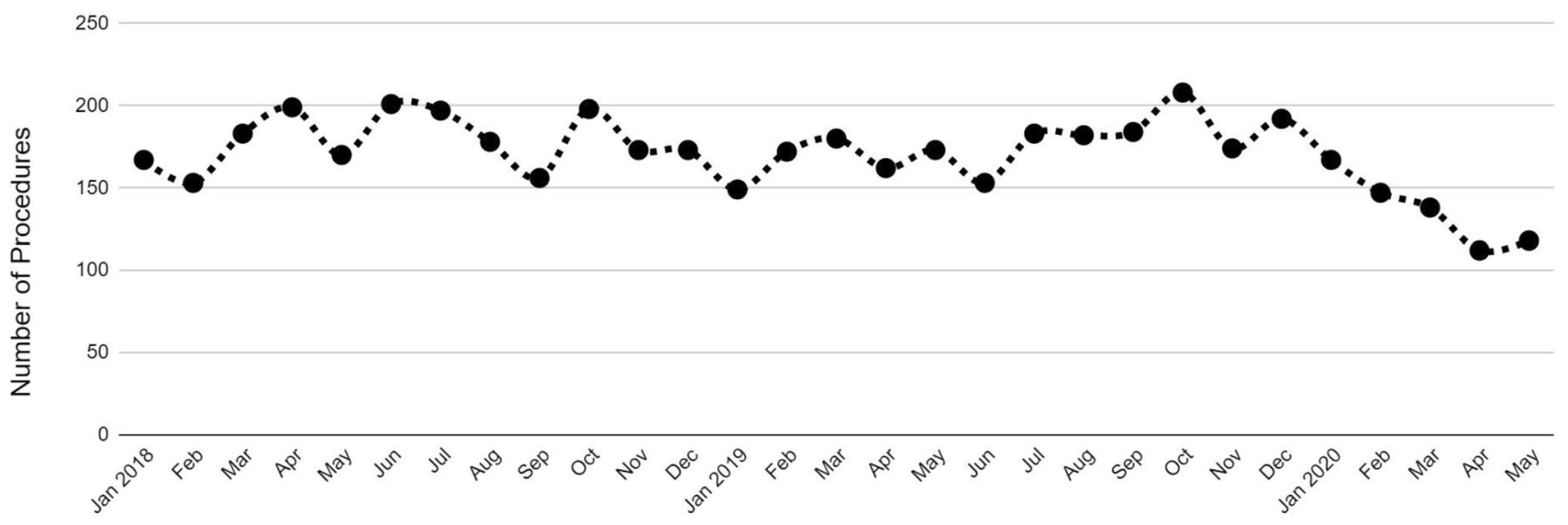

Fig. 1 Total number of procedures per month since January 2018. Note that in previous years there was a drop in the number of procedures in the period December-January (associated to holidays) fol- lowed by an increase in March. However, in 2020 the drop continues to May, setting a new lower monthly rate of procedures 
Table 1 Number of surgeries in each March 1st-May 31st period during 2020 and the previous 5 years

\begin{tabular}{lllllll}
\hline March 1st-May 31st & 2020 & 2019 & 2018 & 2017 & 2016 & 2015 \\
\hline Emergency surgeries & 216 & 222 & 248 & 285 & 201 & 195 \\
Scheduled surgeries & 152 & 293 & 304 & 354 & 284 & 266 \\
Em/Sch ratio & 1.42 & 0.76 & 0.82 & 0.81 & 0.71 & 0.73 \\
Total & 368 & 515 & 552 & 639 & 485 & 461 \\
\hline
\end{tabular}

Surgeries are categorized into two groups: scheduled and emergency. The trend in distribution is expressed through the emergency — scheduled ratio (Em/Sch Ratio)

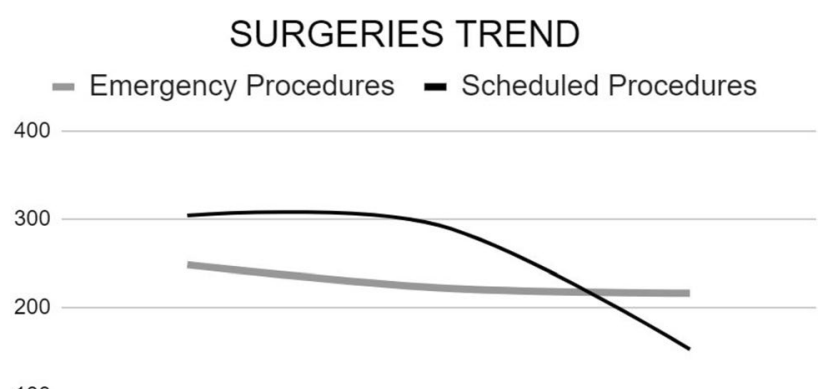

100

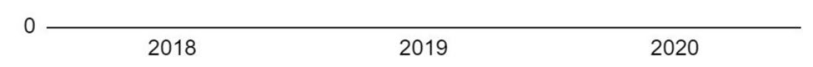

Fig. 2 Number of surgical procedures during the March-May period compared to the previous 2 years. Emergency and scheduled procedures are represented separately. A drop in the scheduled procedures curve (black) is observed in contrast to a stable curve for the number of emergency cases (grey)

To assess changes in the type of surgery pattern, scheduled procedures were categorized into 7 groups, according to the National Surgical Complexity Classification System [5]. We observed that $76 \%(n: 223)$ and $85 \%(n$ : 259) of the scheduled surgeries in 2019 and 2018, respectively, belonged to the III-IV complexity categories. In $2020,75 \%$ ( $n: 114$ ) of the surgeries had III-IV complexity, showing no statistical association in 2020 vs 2019 analysis ( $p$ : 0.934), but correlation in 2020 vs 2018 comparison ( $p$ : 0.011246). Regarding the more complex procedures, categories VI-VII showed an increase in the percentage distribution, and again, with no statistical correlation in $2020(n: 17,11 \%)$ vs. $2019(n: 20,7 \%)$ comparison ( $p$ : $0.107)$ but with a statistical association in the 2020 vs 2018 ( $n: 12,4 \%$ ) comparison ( $p: 0.002647)$. Consequently, a specific change in complexity distribution could not be associated directly with the effect of the Pandemic. However, this disease had a great repercussion on the number of III-IV complexity procedures during $2020(n: 114)$, that showed a $53 \%$ reduction in comparison with the average of the previous 2 years $(n: 241)$.

The surgical residents performed 209 surgical procedures as leading surgeons during the 3 month period in 2020 , which represented $56.8 \%$ of the total number of procedures. We observed a significant reduction in the number and the percentage of procedures performed by the Residents as leading surgeons in 2020 ( $n: 209,56.8 \%)$ compared to 2019 ( $n: 354,68.7 \%, p: 0.000272), 2018$ ( $n$ : $420,76.1 \%, p<0,00001)$ and the average 2018-2019 $(n$ : $387,72.4 \%, p<0,00001)$. The difference between 2019 and 2018 is also relevant but with a weaker statistical association ( $p$ : 0.0072). Factors contributing to that difference should be carefully considered in future studies. Furthermore, the number of surgeries performed by residents in the 2020 trimester ( $n: 209)$ represents a decrease of $46 \%$ when compared to the 2018-2019 average ( $n: 387)$. The mean number of procedures per resident was 20.9 which corresponds to a $56.8 \%$ decrease Table 2 .

Finally, all procedures were performed using the appropriate PPE according to the recommended protocols. There were no COVID-19 infections related to professional activity among residents so far.

\section{The survey}

We collected answers from 10 Pediatric Surgery Residents (4 male and 6 female) who voluntarily participated in the survey ( $100 \%$ response rate). Information is summarized in Table 3.
Table 2 Number of surgical procedures performed by residents acting as leading surgeon in the March-May period

\begin{tabular}{lllll}
\hline March 1st-May 31st & 2020 & 2019 & 2018 & 2018-2019 mean \\
\hline Number of surgeries & 368 & 515 & 552 & 533.5 \\
Procedures performed by residents (\%) & $209(56.8)$ & $354(68.7)$ & $420(76.1)$ & $387(72.4)$ \\
$\begin{array}{l}\text { Procedures per resident (number of active } \\
\text { Residents) }\end{array}$ & $20.9(10)$ & $44.2(8)$ & $52.5(8)$ & 48.4 \\
\hline
\end{tabular}


Table 3 Impact of the COVID 19 pandemic on surgical skills training—self perception survey for the Pediatric Surgery Residency

Item 1: surgical performance

Improvement on surgical performance
Yes

No

Yes

No

Yes

Yes

Hours dedicated to simulation-based laparoscopic training After COVID-19

More than $10 \mathrm{~h}$ a month

(30\% more than $20 \mathrm{~h}$ a month)
$30 \%$

$70 \%$

$50 \%$

$100 \%$

$100 \%$

$100 \%$

$70 \%$

(50\% less than $1 \mathrm{~h}$ a month)

Item 4: level of confidence about surgical skills

Change in confidence during surgery

Decrease in confidence during surgery

No

$30 \%$

Only after weeks of academic duties at home (isolation)

Yes

$70 \%$

$60 \%$

$10 \%$

Item 5: effect of fear of contagion on learning opportunities in the OR

Identified fear of contagion as a limiting factor for learning

No

$80 \%$

Item 6: learning opportunities created by the COVID-19 Pandemic

Participation in complex procedures that would have been performed by a senior year resident

Item 7: preparation for surgery

More available time and used it to study

Achieved better preparation for surgery

Negative impact on preoperative preparation due to loss of face-to-face interaction with staff surgeons or patients prior to surgery

\section{Discussion}

Residency Programs are based on learning through exposure to clinical scenarios in a controlled setting [1]. This is especially important in surgical specialties where the acquisition of skills and abilities requires active guided participation of the residents, and learning is based on daily experience, both in simulation labs and the clinical field [6]. When unexpected external circumstances generate a drastic reduction in the volume of surgeries and procedures, the impact on learning opportunities is unavoidable. Similar to what was reported in other institutions, the restrictions imposed by the COVID-19 pandemic hindered the opportunities of training activities but, in return, created new ones for the development of new teaching strategies [7-10].

The reorganization of the Pediatric Surgery and Urology Division into working teams produced a transient loss of structure and hierarchy of roles among residents. The activities traditionally assigned according to complexity and training objectives were redistributed more homogeneously among the entire work team assigned to each week. Residents of lower years had to face the challenge of rapidly incorporating skills and knowledge that are traditionally more gradually developed and, in return, residents of higher years had to take a more active teaching role, monitoring this learning and its translation into practice. These criteria also applied inside the OR and surgeries were assigned to those on active duty. Quoting T. Dornan et al. "to become capable, learners need to feel psychologically safe. They must not feel too safe, though, because they learn by stepping outside their comfort zones" [6]. However, from the information obtained through the survey, Residents did not perceive a positive effect of the Pandemic in opening opportunities of exposure to more difficult surgical challenges that would be filled by a different resident in normal conditions.

When residents were asked their perception regarding fear of contagion being a conditioning factor for their learning, $80 \%$ did not feel it was the main concern. However, based on additional information obtained through comments registered in the survey, the group recognizes that the need for rapid surgery and the use of PPE could have limited communication during surgery. Some of them even declared a certain "discomfort" or "difficulty" during the procedure creating a less enjoyable environment.

The role of the Chief Resident remained unchanged from a theoretical point of view, but also had to follow the group task schedule. This created additional responsibility for the senior residents who acted as team leaders each week and 
as an interface with both the Chief Resident and the Teaching Coordinator. This change may have a negative effect on residents of lower years, by reducing residents' contact with a strong figure in their daily life and in their training but, at the same time, a positive effect on more advanced residents, by stimulating the progressive acquisition of increasing roles of autonomy and supervision. In our experience, both effects were presented at different time points but were softened through permanent contact and personalized follow-up to each of the residents. This could be a good example of what T. Dornan et al. refer to as "affective support" that a good leader must perform on students, favoring an adequate learning climate [6].

The comparative analysis of the 2020 March 1st-May 31 st period, showed a reduction in the total number of surgeries when compared to the same months of the previous years. Additionally, compared to the last 2 years' average, the total number of surgeries experienced more than a $30 \%$ reduction, with a deeper impact on the scheduled procedures that were cut-off by almost $50 \%$. Surgeries with oncological criteria, the resolution of congenital malformations or urgent neonatal diseases, as well as all those surgeries where the real benefit of its performance outweigh the risks of possible contagion, were maintained [11]. The minimum number of scheduled surgeries in a month that was registered during 2020 was 32 in April, corresponding only to $35 \%$ of the minimum value registered in the previous year. This has certainly an impact on the learning curve, considering that scheduled surgeries constitute the main space for practical teaching interaction between staff physicians, with greater surgical experience, and residents.

Furthermore, lower participation of residents as leading surgeons was observed and the average number of procedures per resident was drastically reduced compared to the previous years' numbers. Additionally, since the residents on-call were not in the hospital, but only came for surgery when a case was presented, the opportunity for preoperative learning related to medical history and diagnosis was lost.

Residents had an abrupt reduction in active working hours and started investing more time in academic activities. However, even though all of them used their "free time" to study, only $60 \%$ admitted to having achieved better preparation for surgery. This probably highlights the importance of clinical settings and "on the job" learning in surgical specialties. For apprentice surgeons, the operating room is the natural environment where the teacher provides the technical basis, and where they apply previously learned tasks, and receive the necessary feedback to complete their learning. Furthermore, this not only translates to surgical skills learning but also affects residents' confidence during surgery as shown in the anonymous survey.

Another loss that was difficult to recover from was the cancellation of the surgical simulation activities that take place regularly in the skills lab. To compensate for the suspension of the in-presence simulation, remote surgical simulation was introduced through a virtual platform. This new teaching methodology could be implemented thanks to the collaboration between residents and staff. All the residents reported participation in simulation training activities during the analyzed period with a marked increase in the number of hours dedicated to laparoscopic training as shown in the survey. The increased number of hours at home, the need to compensate for the reduction in OR activity, and the intrinsic motivation of the residents and teachers, allowed establishing an alliance through which they adapted to the new circumstances. This active role in the construction of learning had also a positive effect on the commitment to activities. This has been a great practical example of the value of information and communication technologies (ICTs) as mediating instruments of the relationships between students and learning contents $[10,12,13]$

We must emphasize that the measures implemented, allowed that no cases of COVID-19 related to Residency activities have been registered among residents so far. Given that the pandemic is currently unfolding in our city, its final impact on the training of residents cannot yet be quantified. As long as the current situation remains active, coordinated efforts must be made to guarantee the fulfillment of the training objectives, without putting the health of the residents at risk and ensuring the best quality of care for our patients. Further analysis will be needed to assess the final impact of these "lost learning opportunities" on surgical skills and residents' confidence.

\section{Limitations}

We recognize that the present study only addresses the impact of the pandemic over a single surgical residency program. Even though its results may not be entirely applicable to other centers (with different settings), it provides useful guiding information and awareness.

\section{Conclusions}

Experienced-based learning is a key element for surgical disciplines. In that context, the COVID-19 pandemic had a major impact on pediatric surgery residents' training by drastically reducing their exposure to OR learning opportunities. Additionally, even though it has favored the development of new teaching strategies such as the introduction of remote simulation-based training activities, residents still perceive a decrease in their surgical confidence that could potentially affect their quality of performance. 
Supplementary Information The online version contains supplementary material available at https://doi.org/10.1007/s00383-021-04961-8.

Acknowledgements To Amanda R. Soto for her valuable suggestions and contributions.

Author contributions All authors contributed to the study conception and design. Material preparation, data collection, and analysis were performed by RB, AC, CP and LL. The first draft of the manuscript was written by RB, CP, LL and PL. All authors made a substantial contribution and commented on previous versions of the manuscript. All authors read and approved the final manuscript and agree to be accountable for all aspects of the work.

\section{Declarations}

Conflict of interest We have no potential competing interest (nor financial or personal) to disclaim.

\section{References}

1. Durante E (2012) La enseñanza en el ambiente clínico: principios y métodos. Revista de Docencia Universitaria 10:149-175

2. Clinical guide for the management of paediatric patients during the coronavirus pandemic(2020). Specialty guides for patient management during the Coronavirus Pandemic. NHS. https:// www.networks.nhs.uk/nhs-networks/south-thames-paediatricnetwork/coronavirus-advice-and-guidance/clinical-guide-for-themanagement-of-paediatric-patients-duringthe-coronavirus-pande mic. Accessed Apr 2020

3. Leva E, Morandi A, Sartori A, Macchini F, Berrettini A, Manzonib G (2020) Correspondence from Northern Italy about our experience with COVID-19. J Pediatr Surg 55(5):985-986

4. Procedimiento de Realización de cirugías de urgencia en pacientes confirmados o con sospecha de COVID-19 (2020). Hospital Italiano de Buenos Aires. https://hiba.hospitalitaliano.org.ar/ intranet/covid19/index.php?contenido=ver_curso.php\&id_curso= 106062. Accessed 1 Apr 2020
5. Nomenclador Nacional de Cirugía Pediátrica (2020). Asociación Civil Argentina de Cirugía Pediátrica (ACACIP). Updated in October. https://www.acacip.org.ar/docs/NOMENCLADOROctubre-2020. Accessed Oct 2020

6. Dornan T, Conn R, Monaghan H et al (2019) Experience based learning (ExBL): clinical teaching for the twenty-first century. Med Teach 41(10):1098-1105

7. Nassar AH et al (2020) Emergency restructuring of a general surgery residency program during the coronavirus disease 2019 pandemic - the University of Washington experience. JAMA Surg. https://doi.org/10.1001/jamasurg.2020.1219

8. Osama M et al (2020) Impact of COVID-19 on surgical residency programs in Pakistan; a residents' perspective. Do programs need formal restructuring to adjust with the "new normal"? A crosssectional survey study. Int J Surg 79:252-256

9. Rosen GH, Murray KS, Greene KL, Pruthi RS, Richstone L, Mirza M (2020) Effect of COVID-19 on urology residency training: a nationwide survey of program directors by the Society of Academic Urologists. J Urol 204:1039-1045

10. El Hawary H, Salimi A, Alam P, Gilardino MS (2020) Educational alternatives for the maintenance of educational competencies in surgical training programs affected by the COVID-19 pandemic. J Med Educ Curric Dev 7:1-3

11. Clinical guide for surgical priorisation during the Coronavirus Pandemic (2020). Specialty guides for patient management during the Coronavirus Pandemic; FSSA. https://www.rcseng.ac.uk/ coronavirus/surgical-prioritisation-guidance. Accessed Apr 2020

12. Coll C (2009). Aprender y enseñar con las TIC: expectativas, realidad y potencialidades. Included in: Carneiro R, Toscano J.C y Díaz T, Los desafíos de las TIC para el cambio educativo. Madrid (Spain): OEI-Santillana, Fundación Santillana. 113-126

13. Porpiglia F, Checcucci E, Amparore D, Verri P, Campi R, Claps F et al (2020) Slowdown of urology residents' learning curve during the COVID-19 emergency. BJU Int 125(6):15-17

Publisher's Note Springer Nature remains neutral with regard to jurisdictional claims in published maps and institutional affiliations. 